\title{
Singular superposition elastostatics BEM/GA algorithm for cavity detection
}

\author{
D. Ojeda ${ }^{1,4}$, B. Gámez ${ }^{1,4}$, E. Divo ${ }^{2}$, A. Kassab ${ }^{3}$ \& M. Cerrolaza ${ }^{4}$ \\ ${ }^{1}$ Department of Mechanical Design and Automation, \\ University of Carabobo, Venezuela \\ ${ }^{2}$ Department of Engineering Technology, \\ University of Central Florida, USA \\ ${ }^{3}$ Department of Mechanical, Materials, and Aerospace Engineering, \\ University of Central Florida, USA \\ ${ }_{4}^{4}$ National Institute of Bioengineering, \\ Central University of Venezuela, Venezuela
}

\begin{abstract}
A method for the efficient solution of the inverse geometric problem for cavity detection using a point load superposition technique in the elastostatics boundary element method (BEM) is presented in this paper. The superposition of point load clusters to simulate the presence of cavities offers tremendous advantages in reducing the computational time for the elastostatics field solution as no boundary re-discretization is necessary throughout the inverse problem solution process. The inverse solution is achieved in two steps: (1) fixing the location and strengths of the point loads, (2) locating the cavity(ies) geometry(ies). For a current estimated point load distribution, a first objective function measures the difference between BEM-computed and measured deformations at the measuring points. A Genetic Algorithm (GA) is employed to automatically alter the locations and strengths of the point sources to minimize the objective function. The GA is parallelized and dynamically balanced. Upon convergence, a second objective function is defined and minimized to locate the cavity(ies) location(s) indicated as traction-free surface(s). Results of cavity detection simulations using numerical experiments and simulated random measurement errors validate the approach in regular and irregular geometrical configurations with single and multiple cavities. Keywords: boundary element method (BEM), cavity detection, genetic algorithm, elastostatics.
\end{abstract}




\section{Introduction}

The purpose of solving most inverse problem is to find the unknown: (a) in the governing equation for field variable, (b) physical properties, (c) boundary conditions, (d) initial condition(s), or (e) the system geometry using over-specified conditions. Typically, the over-specified conditions are provided by measuring a field variable at the exposed boundary, as in the case of the inverse geometric problem. However, in some inverse problems, the over-specified condition can be provided by internal measurements of field variable via embedded sensors (Divo et al [1], Ulrich et al [2], Kassab et al [3]). In this paper, such measurements, along with accompanying noise, are simulated numerically. The purpose of the inverse geometric problem, that concerns this study, is to determine the hidden portion of the system geometry by using over-specified boundary conditions on the exposed portion. This problem has gained importance in thermal and solid mechanics applications for nondestructive detection of subsurface cavities (Ulrich et al [2], Kassab et al [3, 4], Divo et al [5]). In thermal applications, the method requires over-specified boundary conditions at the surface, i.e., both temperature and flux must be given, Divo et al [1]. In elastostatics applications, the over-specified conditions are provided in terms of surface displacements and tractions. Generally, surface tractions are known boundary conditions, while the surface displacements are experimentally determined by measurements, (see Ulrich et al [2], Kassab et al [3]).

A variety of numerical methods have been used to solve the inverse geometric problem. This inverse problem has applications in the identification of surfaces flaws and cavities and in shape optimization problems (Divo et al [1, 5, 79], Ulrich et al [2], Kassab et al [3, 4], Bialecki et al [6]). The computational burden is intensive due to the inherent nature of the solution of inverse problem which requires numerous forward problems to be solved, regardless of whether a numerical or analytical approach is taken to solve the associated direct problem. Moreover, in the inverse geometric problem, a complete regeneration of the mesh is also necessary as the geometry evolves. Boundary Element Methods (BEM) lends themselves naturally to the numerical solution of the inverse problem (see Divo et al [1], Ulrich et al [2], Kassab et al [3], Cerrolaza et al [10], Müller-Karger et al [11], Annicchiarico et al [12-14], and Martinez and Cerrolaza [15]) and this is because the solution algorithms developed by researchers typically involve minimization of residuals, which measure the non-satisfaction of over-specified boundary conditions. Additionally, in the iterative solution of this problem the geometry is continuously updated. This places a premium on a numerical method, which does not require domain discretization (Brebbia and Dominguez [16]).

A method for the efficient solution of the inverse optimization problem of cavity detection using a point load superposition technique in elastostatics boundary element methods is presented in this paper. The superposition of point load clusters in the domain is posed as an alternative to satisfy the Cauchy conditions on the surface. The point loads must be located inside the eventual cavity or outside the domain in order to correctly satisfy the governing equation. Using 
Genetics Algorithms, the point load distribution, strength, and location are altered to seek satisfaction of the over specified boundary displacements. Numerical results of direct 2D problems using the BEM are used as an alternative to validate the approach. Results of cavity detection problems simulated using numerical experiments and added random measurement errors validate the approach in regular and irregular geometrical configurations with single and multiple cavities.

\section{Direct problem and BEM in elasticity}

The solution of the forward elastostatics problem is expressed in terms of displacements which, for an isotropic, homogeneous, and linearly elastic medium imposed with an internal volumetric force $b_{i}$, is governed by Navier's equation as:

$$
\mu \frac{\partial^{2} u_{i}}{\partial x_{i} \partial x_{j}}+\left(\frac{\mu}{1-2 \nu}\right) \frac{\partial^{2} u_{j}}{\partial x_{i} \partial x_{j}}+b_{i}=0
$$

Here, $\underset{\sim}{u}$ is the displacement vector, $\nu$ is Poisson's ratio, and $\mu$ is shear modulus. Introducing the fundamental solution to Navier's equation, a BEM formulation can be derived from the Somigliana identity providing an integral relation between the displacement vector $u_{i}^{p}$ in a point collocation " $p$ " and displacement vectors $u_{i}$ and traction vectors $t_{i}$ at the boundary $\Gamma$ as well as the body forces $b_{i}$ :

$$
c_{i j}^{p} u_{i}^{p}+\oint_{\Gamma} H_{i j} u_{i} d \Gamma=\oint_{\Gamma} G_{i j} t_{i} d \Gamma+\int_{\Omega} G_{i j} b_{i} d \Omega
$$

Here, $\Omega$ is the problem domain, $G_{i j}$ and $H_{i j}$ are the displacement and traction fundamental solutions (see Brebbia et al [16]). Establishing that internal force $b_{i}$ is formed only by points loads, so that:

$$
b_{i}=\sum_{l=1}^{N L} Q_{i}^{l} \delta\left(x_{i}, x_{i}^{l}\right)
$$

where $N L$ is the number of points loads, $Q_{i}^{l}$ is the intensity of each load and $\delta\left(x_{i}, x_{i}^{l}\right)$ is the Dirac's delta function located in the impact point of each load $x_{i}^{l}$. Using the properties of the Dirac's delta function, the last integral equation term Eq. (2) is reduced to:

$$
c_{i j}^{p} u_{i}^{p}+\oint_{\Gamma} H_{i j} u_{i} d \Gamma=\oint_{\Gamma} G_{i j} t_{i} d \Gamma+\sum_{l=1}^{N L} Q_{i}^{l} G_{i j}^{l}
$$

Employing standard boundary element procedures, the above equation is written in discrete form as:

$$
[H]\{u\}=[G]\{t\}+\{q\}
$$

where the matrices $[H]$ and $[G]$, with dimensions $N \times N$, contain the influence coefficient that relate displacement and traction vectors $\{u\}$ and $\{t\}$ on the 


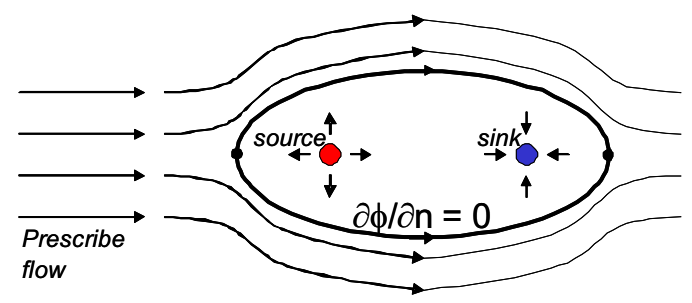

Figure 1: Simulation of elliptical surface with an incoming parallel flow by singularities superposition.

boundary. The size of $N$ is $N=d \times N E \times N N$, where, $d$ is the number of space dimensions (2 or 3), $N E$ is the number of elements, and $N N$ is the number of nodes per element. It is worth noting that all effects generated by points loads are located in the vector $\{q\}$, therefore, when point loads that are utilized in the inverse geometric problem solution are relocated in the evolving solution, there is no need for boundary remeshing.

Introducing the boundary condition $\overline{u_{i}}$ and $\overline{t_{i}}$ in Eq. (5), an algebraic system with the following form is obtained: $[A]\{x\}=\{b\}+\{q\}$. The vector $\{x\}$ contains the unknowns values of $\{u\}$ and $\{t\}$. This system of equations is solved using a standard method. In this paper, we use isoparametric-discontinuous-quadratic elements: the geometry and vectors $\{u\}$ and $\{t\}$ values are approximated using quadratic shape function locating the displacement and traction nodes within the element boundaries.

\section{Cavity simulation with point loads}

The approach proposed in this paper for the modeling of internal cavity(ies) is inspired from potential theory. For example, the superposition of a source and a sink with the same strength located a distance $L$ in a prescribed parallel flow results in iso-flow lines and simulate the presence of a solid surface through the iso-lines containing stagnation points. This null-flow line can be interpreted as the presence of a solid surface or the artificial contour of an elliptical cavity, see Figure 1. The notion of utilizing point sources and sinks to model cavities has been successfully utilized by Divo et al [1] in solving the inverse geometric problem by thermal methods. This theory can be applied in elastostatics field where the interpretation is understood as superposition of point loads and the flow is considered to be that of elastic energy. With proper adjustment of the location, number, and intensities of these loads, one can generate a surface (or surfaces) that are traction-free and therefore interpreted as a cavity surface(s), this is illustrated in Figure 2, where a cluster of such point loads is shown. 


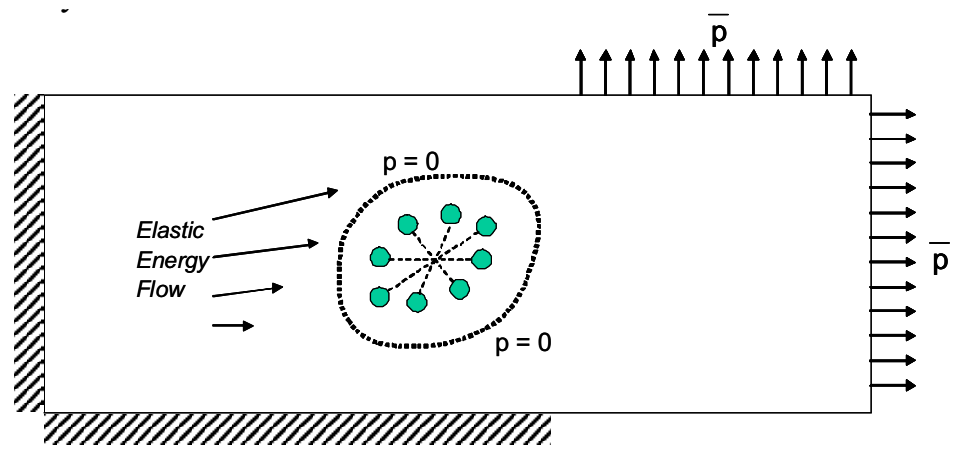

Figure 2: Geometric arrangement of points loads with a non-uniform elastic energy flow.

\section{Inverse problem and objective function}

The numerical inverse process for cavity detection is achieved in 2 steps: (1) fixing the location and strengths of the fictitious point loads, (2) locating the cavity(ies) geometry(ies). For a current estimated point load distribution, a first objective function measures the difference between BEM-computed and measured deformations at the measuring points. Since the governing equation for the elasticity problem is the Navier Equation without body forces, the fictitious point loads must be located outside the problem domain, that is outside the exposed bounding surface or within the subsurface cavities. As such, the first iteration process searches for locations and strengths of the fictitious point loads until a match is found between the tractions and deformations computed by the BEM and those measured on the boundary as additional information or over-specified conditions. This is achieved by the minimization of an objective function, $S 1$, that quantifies the difference between the deformations $u_{i}$ obtained by BEM (Eq. (5)) and measured deformations $\widehat{u}_{i}$ providing the additional information obtained through experimental measurements on the exposed boundaries (see Divo et al [1], Ulrich et al [2], Kassab et al [3, 4]). Upon convergence, a second objective function is defined and minimized to locate the cavity(ies) location(s) indicated as traction-free surface(s). A Genetic Algorithm (GA) is employed to solve both minimization problems and it is parallelized and dynamically balanced.

\section{Method of optimization: GA}

The GA used for this optimization process models the objective function as a haploid with a binary vector to model a single chromosome as described in Divo et al [1]. The length of the vector is dictated by the number of design variables and the required precision of each design variable. Each design variable has to be bounded with a minimum and a maximum value, and in the process the precision 


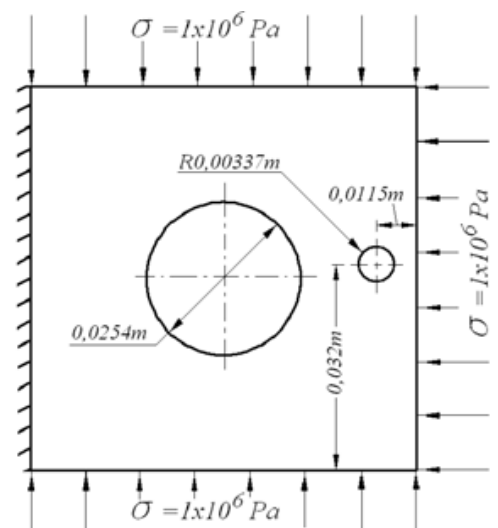

(a) Boundary conditions.

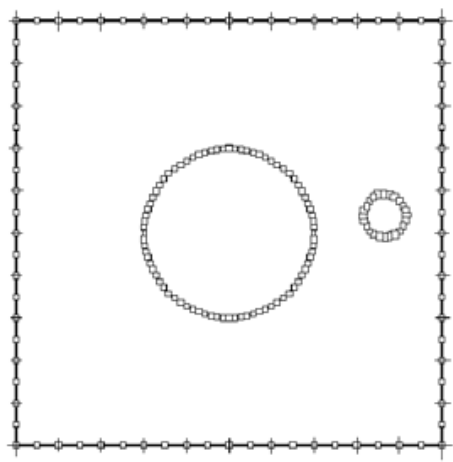

(b) Discretization: 80 elements.

Figure 3: A square plate with centered circular hole of diameter $0.0254 \mathrm{~m}$.

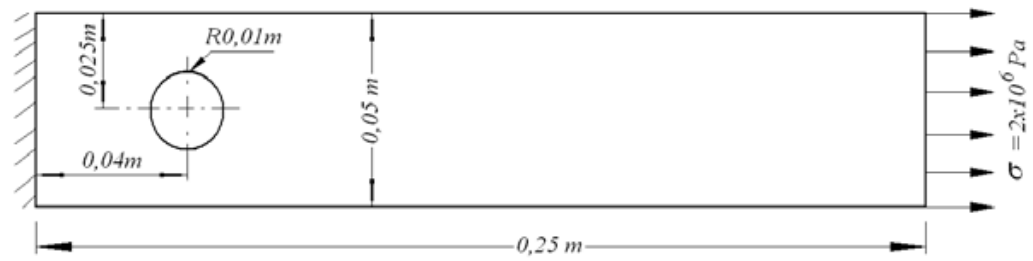

Figure 4: An elongated rectangular bar with a circular cavity under tension.

of the variable is determined. This procedure allows an easy mapping from real numbers to binary strings and vice versa. This coding process represented by a binary string is one of the distinguishing features of GA and differentiates them from other evolutionary approaches. The haploid GA place all design variables into one binary string, called a chromosome or offspring. The GA optimization process begins by setting a random set of possible solutions. Each individual is defined by parameters combinations, which in this case are $\left(Q_{i}^{l}, x_{c}, y_{c}, r_{x}, r_{y}, \theta\right)$ and is represented as a bit string or a chromosome. Since GA are used to maximize and not minimize, an aptitude function, $Z$, is formulated as the inverse of the objective function. This aptitude function $Z$ is evaluated for every individual in the current population defining the fitness or their probability of survival. A series of parameters are initially set in the GA code, and these determine and affect the performance of the genetic optimization process. 


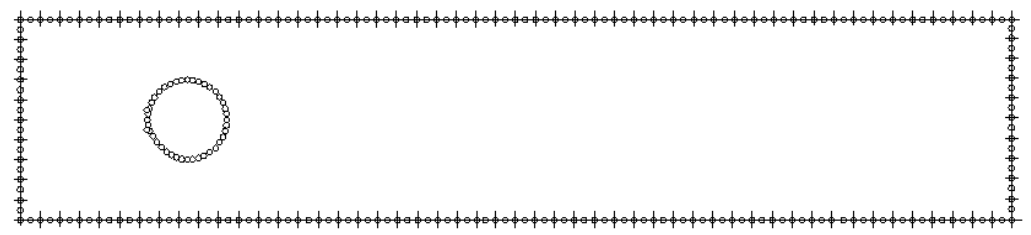

Figure 5: Discretization of the rectangular bar under tension using 120 elements.
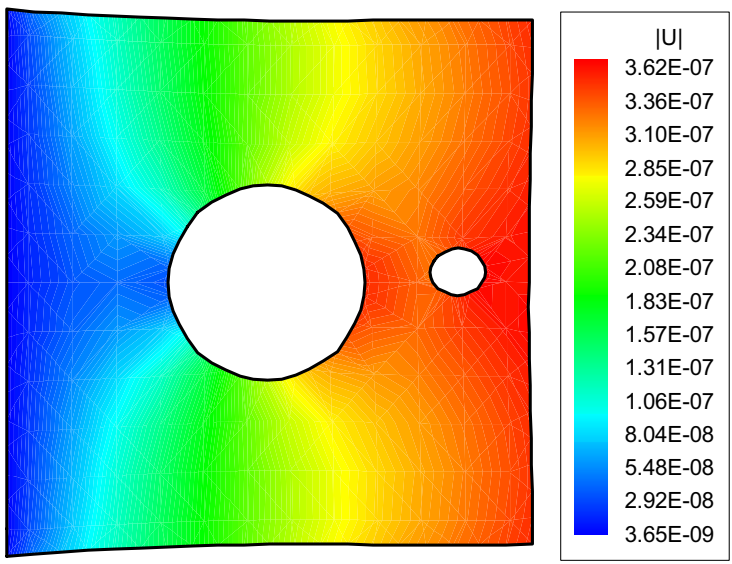

(a) Example 1.

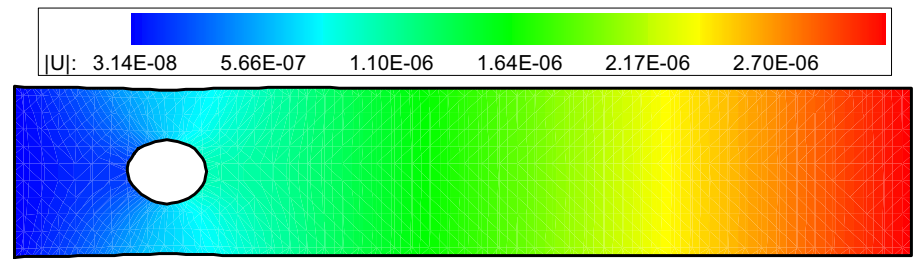

(c) Example 2 .

Figure 6: Contour of BEM-computed displacements, in $[m]$.

\section{Numerical examples}

Results obtained from solving forward problems are used to generate the displacement results at the external surface to simulate experimental measurements. The latter are ladened with random error to simulate noise. Both forward and inverse problems use the BEM as the field solver. The BEM model uses discontinuous quadratic elements with adaptive quadratures. The first example, displayed in Figure 3, considers a $0.0635 \times 0.0635 \mathrm{~m}^{2}$ square plate with a $0.0254 \mathrm{~m}$ diameter centered hole and a $0.00674 \mathrm{~m}$ diameter cavity. The plate is 


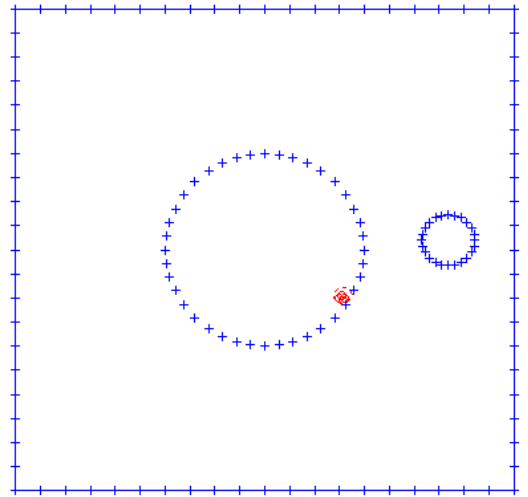

(a) $2^{\text {nd }}$ generation

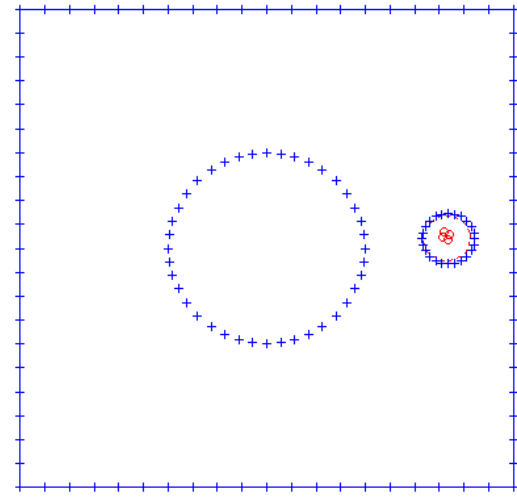

(b) $2600^{\text {th }}$ generation

Figure 7: Evolution of cavity detection for example.

clamped in one side and the others are imposed with a $10^{6} \mathrm{~Pa}$ uniform compression loads. The second example is displayed in Figure 4: a rectangular $0.25 \times 0.05 \mathrm{~m}^{2}$ clamped bar with a uniform $2 \times 10^{6} \mathrm{~Pa}$ imposed traction load. The problem is discretized with 120 elements. The bar has a $0.02 m$ diameter cavity located $0.4 m$ from the left-end of the bar, see Figure 5. The displacements for the two forward problems shown in Figures 6(a) and 6(b) are used as inputs for the inverse problem.

The computed surface displacements at the traction-free exposed surfaces in both of the problems are used as additional information to solve the inverse geometric problem of cavity detection, and, in addition, a random error of $\pm 1 \times 10^{-8} \mathrm{~m}$ is added to these surface displacements to mimic measurement error. The location of the cluster of singularities along with the strengths and cluster evolution, are shown in Figures 7(a) and 7(b) for the first example. Similarly, these are displayed in Figures 8(a) and 8(b) for the second example. The evolutions of the first and second objective functions for Example 1 are shown in Figures 9(a) and 9(b). In these two examples, the approach proposed in this paper is demonstrated to be successful in locating subsurface cavities using an inverse elastostatics BEM-point load superposition method.

\section{Conclusions}

A method for the efficient solution of the inverse optimization problem of cavity detection using a point load superposition technique in elastostatics boundary element methods is development in this paper. Two examples demonstrate the ability of the method to successfully locate single cavities in terms of their locations and size whilst using inputs ladened with simulated random error. The GA has been integrated as optimization tool using BEM. The technique is readily applicable to the closely related problem of shape optimization, in which the condition at the cavity side may be arbitrarily specified as a design target. 


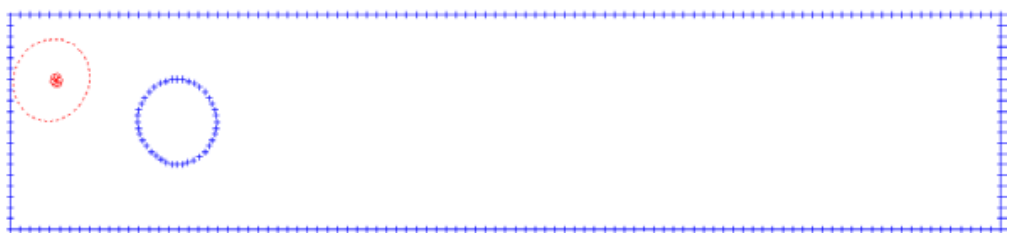

(a) $2^{\text {nd }}$ generation

(b) $3200^{\text {th }}$ generation

Figure 8: Evolution of cavity detection for example 2.

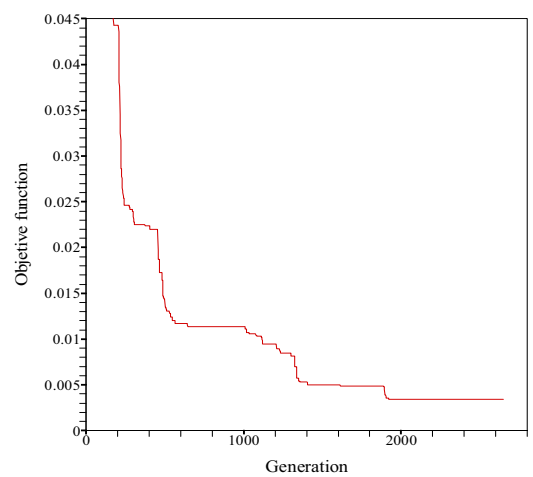

(a) $1^{\text {st }}$ Objective function evolution.

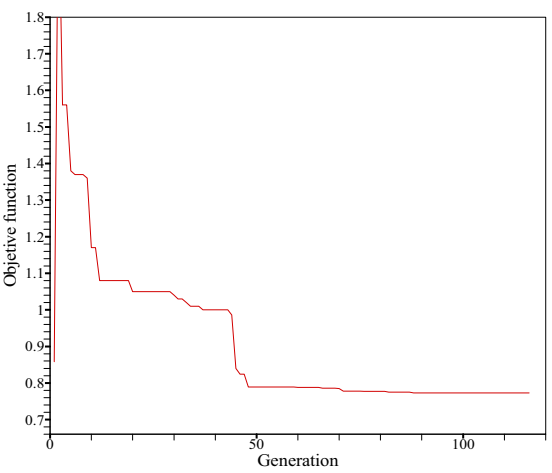

(b) $2^{\text {nd }}$ Objective function evolution.

Figure 9: Objective function evolution for example 1.

\section{Acknowledgements}

The work undertaken in this project was carried out under the institutional and financial support provided by the University of Central Florida (USA), the University of Carabobo (Venezuela), and FONACIT (Venezuela).

\section{References}

[1] Divo E.A., Kassab A.J., Rodríguez F., An efficient singular superposition technique for cavity detection and shape optimization. Numerical Heat Transfer, Part B, 46: 1-30, 2004, Copyright Taylor \& Francis Inc.

[2] Ulrich T.W., Moslehy F.A. and Kassab A.J., A BEM based pattern search solution for a class of inverse elastostatic problems. Int. J. Solids Structures, Vol. 33, No. 15, pp. 2123-2131, 1996, Copyright 1996. 
[3] Kassab A.J., Moslehy F.A., Daryapurkar A.B., Nondestructive detection of cavities by an inverse elastostatics boundary element method. J. Engineering Analysis with Boundary Elements 13 (1994) 45-55.

[4] Kassab A.J., Moslehy F.A., Ulrich T.W., Inverse boundary element solution for locating subsurface cavities in thermal and elastostatic problems. In Proc. IABEM-95, Computational Mechanics '95, Hawaii, July 30-August 3 (ed. Atluri, Yagawa and Cruse), pp. 3024-3029, Springer, Berlin.

[5] Divo E.A., Kassab A.J., Rodríguez F., Characterization of space dependent thermal conductivity with a BEM-Based genetic algorithm. Institute for Computational Engineering, University of Central Florida, Orlando, Florida, 32816-2450, Numerical Heat Transfer, Part A: Applications, Vol. 37, No. 8, (2000), pp. 845-877.

[6] Bialecki R. Divo E. Kassab A., Reconstruction of time-dependent boundary heat flux by a BEM-based inverse algorithm. J. Engineering Analysis with Boundary Elements 30 (2006) 767-773.

[7] Divo E.A., Kassab A.J., A meshless method for conjugate heat transfer problems. J. Eng. Analysis with Boundary Elements 29 (2005) 136-149.

[8] Divo E.A., Kassab A.J., Ingber M.S., Shape optimization of acoustic scattering bodies. J. Engineering Analysis with Boundary Elements 27 (2003), 695-703.

[9] Divo E.A., Kassab A.J., Kapat J.S., Chyu Ming-King, Retrieval of multidimensional heat transfer coefficient distributions using an inverse BEM-based regularized algorithm: numerical and experimental results. $J$. Engineering Analysis with Boundary Elements 29 (2005), 150-160.

[10] Cerrolaza M., Annicchiarico W. and Martinez M., Optimization of 2D boundary element models using $\beta$-splines and genetic algorithms, Engineering Anal. with Bound. Elem., 24(5): (2000), 427-440.

[11] Müller-Karger C., González C., Aliabadi M.H. and Cerrolaza M., Three dimensional BEM and FEM stress analysis of the human tibia under pathological conditions, J. of Comp. Mod. In Eng. and Sciences, 2(1): (2001), $1-13$.

[12] Annicchiarico W. And Cerrolaza M., An Evolutionary Approach for the Shape Optimization of General Boundary Elements Models. Electronic Journal of Boundary Elements, Vol.2, (2002)

[13] Annicchiarico W. and Cerrolaza M., A 3D boundary element optimization approach based on genetic algorithms and surface modeling, Eng. Anal. With Bound. Elem., Vol. 28(11), (2004), pp. 1351-1361

[14] Annicchiarico W., Martínez G., Cerrolaza M., Boundary elements and $\beta$ spline modeling for medical applications, J. of App. Math. Mod., (2005), (in press)

[15] Martínez G. and Cerrolaza M., A bone adaptation integrated approach using BEM, J. Eng. Anal. With Bound. Elem. 30, (2006), 107-115

[16] Brebbia C.A., Dominguez J., Boundary element, An introductory course. Computational Mechanics Publ., pp. 134-250, Boston, co-published with McGraw-Hill, New York, 1989. 